\title{
Optical coupling method utilizing a lensed fiber integrated with a long-period fiber grating
}

\author{
Wen Tzung Chen and Lon A. Wang
}

\begin{abstract}
A novel optical coupling scheme that uses a lensed fiber integrated with a long-period fiber grating (LPFG) is proposed. Two experiments are performed to demonstrate the validity of such a scheme in single-mode-fiber-single-mode-fiber (SMF-to-SMF) and laser-diode-single-mode-fiber (LD-to-SMF) coupling setups. The measured results show that for an appropriate lens radius the addition of a LPFG will lead to a higher coupling efficiency over a longer range of working distance than without the LPFG. Coupling efficiencies of $\sim 78 \%$ and $35 \%$ are achieved for corresponding working distances of $\sim 250$ and 110 $\mu \mathrm{m}, 1-\mathrm{dB}$ longitudinal tolerances of $\sim 40$ and $26 \mu \mathrm{m}$, and 1-dB transverse tolerances of $\sim 7.6$ and $2.6 \mu \mathrm{m}$ for SMF-to-SMF and LD-to-SMF, respectively. (C) 2000 Optical Society of America

OCIS codes: $060.2310,050.2770$.
\end{abstract}

\section{Introduction}

Recently, long-period fiber gratings (LPFG's) have been used in various applications such as bandrejection filters, gain equalizers, rocking filters, mode converters, and sensors. ${ }^{1-5}$ Here we propose a new application in which a LPFG and an integrated hemispherical lensed fiber are used to assist optical coupling.

Various schemes that use lensed fibers have been reported in the past few years for applications of single-mode-fiber-single-mode fiber (SMF-toSMF) and laser-diode-single-mode-fiber (LD-toSMF) coupling. ${ }^{6-9}$ Practically, a lensed fiber has many desirable features, such as high coupling efficiency, compactness, mechanical stability, and economical performance. However, a lensed fiber also has several disadvantages, such as a small working distance (roughly shorter than its core diameter, $\sim 10 \mu \mathrm{m})$, tight transverse tolerance $(\sim 0.3$ $\mu \mathrm{m}$ for $1-\mathrm{dB}$ loss), and critical constraints on the fabrication process (lens radius, $\cong 5 \mu \mathrm{m}$ ). A working distance longer than $\sim 100 \mu \mathrm{m}$ is generally desired for LD-to-SMF packaging of an optical subassembly module ${ }^{10}$ and for the reduction of op-

The authors are with the Department of Electrical Engineering and Institute of Electro-Optical Engineering, National Taiwan University, Taipei, Taiwan. L. A. Wang's e-mail address is lon@ccms.ntu.edu.tw.

Received 14 January 2000.

0003-6935/00/254490-11 $\$ 15.00 / 0$

(C) 2000 Optical Society of America tical backreflection. For example, in the case of lensed fiber with a $50-\mu \mathrm{m}$ radius, the optical backreflection decreases from -30 to $-60 \mathrm{~dB}$ when the working distance increases from 10 to $100 \mu \mathrm{m} .{ }^{11}$ Although it has been reported that an integrated spot size converter improves coupling, ${ }^{12}$ the working distance is typically not more than $20 \mu \mathrm{m}$; thus higher optical backreflections can be induced for an uncoated and cleaved fiber. Various approaches to increasing the working distance have been tried, but most working distances are still limited to less than $50 \mu \mathrm{m} \cdot{ }^{13,14}$ Although the use of thermally expanded core fiber integrated with a coreless fiber could lead to a larger working distance, the fabrication processes with this type of fiber are relatively time consuming and complicated. ${ }^{10}$ For example, the fabrication of a thermally expanded fiber may require $1 \mathrm{~h}$ at $1700{ }^{\circ} \mathrm{C}$, and three different fibers may be needed for integration.

To overcome these disadvantages, we propose the novel coupling scheme shown schematically in Fig. 1, in which ray optics is used for qualitative analysis. In Section 2 we present a more rigorous analysis. A portion of the rays coming from a finite source is coupled into the cladding of a receiving single-mode fiber, SMF1, by a hemispherical lensed fiber. These rays pass through the LPFG in region III and diffract into different orders. The diffracted rays that satisfy the total internal reflection condition

$$
\theta_{4}=\sin ^{-1}\left\{\frac{1}{n_{\mathrm{co}}}\left[n_{\mathrm{cl}} \sin \theta_{3}+m\left(\frac{\lambda}{\Lambda}\right)\right]\right\}>\sin ^{-1}\left(\frac{n_{\mathrm{cl}}}{n_{\mathrm{co}}}\right)
$$




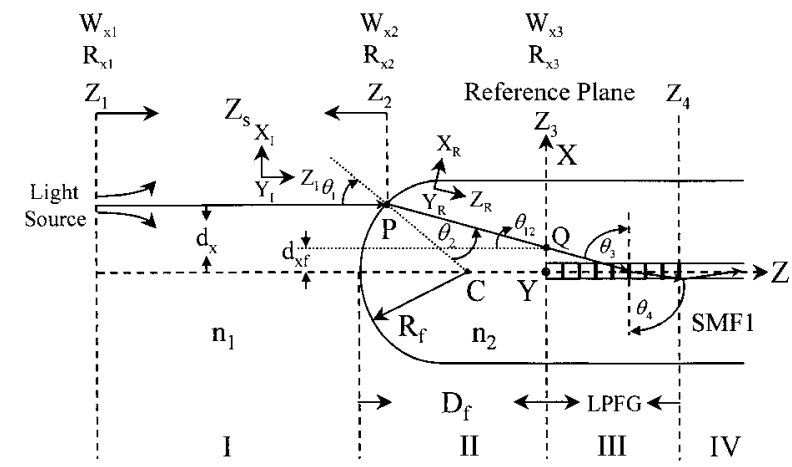

Fig. 1. Schematic diagram of the proposed novel optical coupling scheme. Abbreviations are defined throughout the text.

remain propagating in the core. Here $\theta_{3}$ and $\theta_{4}$ are the incident and diffraction angles, respectively; $m$ is an integer, $\Lambda$ is the period of the LPFG; and $n_{\text {co }}$ and $n_{\mathrm{cl}}$ are the refractive indices in the core and the cladding, respectively. The other rays received by the core of SMF1 are coupled into the cladding because of the presence of the LPFG.

As we reported previously, ${ }^{15}$ the main advantage of using such a coupling scheme is the resultant long working distance because the launched beam is coupled to the relatively large cladding area relative to the core area. Although the LPFG plays a vital role in assisting coupling, fabrication of the LPFG is simple. ${ }^{16-19}$ The radius of the lensed fiber used in this coupling scheme is larger than that of a conventional fiber, which permits the fabrication tolerances to be relaxed. Therefore the proposed lensed fiber assisted by a LPFG may serve as a useful coupling device in the packaging of an optical subassembly.

The remainder of this paper is arranged as follows: In Section 2 we use a mode-expansion method, including core and cladding modes, to calculate the coupling efficiency. In Section 3 two we demonstrate proofs of experiment to show the validity of the proposed scheme. In Section 4 we first discuss the differences between the measured and simulated results and then analyze the optimal working distance and lens radius for the proposed scheme. Moreover, we discuss some practical applications of the scheme. Finally, we conclude our study in Section 5.

\section{Principle}

First we use an $A B C D$ matrix to locate a Gaussian beam and then we apply the mode-expansion method to calculate the coupling efficiency of a Gaussian beam and a fiber cladding mode. As shown in Fig. 1, a Gaussian beam initially located at plane $\mathrm{Z}_{1}$ has a beam waist $W_{x 1}=W_{0}$, a radius of curvature $R_{x 1}=\infty$, and a transverse displacement $d_{x}$. After the beam has propagated a distance $Z_{s}$ in free space, the new waist $W_{x 2}$ and radius of curvature $R_{x 2}$ at plane $\mathrm{Z}_{2}$ can be expressed as

$$
\begin{aligned}
& W_{x 2}=W_{0}\left[1+\left(\frac{Z_{s}}{Z_{0}}\right)^{2}\right]^{1 / 2}, \\
& R_{x 2}=Z_{s}\left[1+\left(\frac{Z_{0}}{Z_{s}}\right)^{2}\right],
\end{aligned}
$$

where $Z_{0}=n_{1} \pi W_{0}^{2} / \lambda_{0}$, and $n_{1}$ is the refractive index of region I.

The Gaussian beam is incident upon the lensed fiber at an angle $\theta_{1}$ owing to a transverse offset. To match the boundary conditions of the complex phases for the incident and refracted beams at point $\mathrm{P}$ we have

$$
\phi_{1}\left(x_{1}, y_{1}, z_{1}\right)=\phi_{R}\left(x_{R}, y_{R}, z_{R}\right)
$$

where

$$
\begin{gathered}
\phi_{i}\left(x_{i}, y_{i}, z_{i}\right)=k_{i} z_{i}+\frac{k_{i}}{2}\left(\frac{x_{i}}{q_{x i}}+\frac{y_{i}}{q_{y i}}\right), \\
\frac{1}{q_{i}}=\frac{1}{R_{i}}-j \frac{\lambda_{0}}{n_{i} \pi W_{i}^{2}}, \quad k_{i}=2 \pi n_{i} / \lambda_{0},
\end{gathered}
$$

$i=I, R$ correspond to incident and refracted beams, respectively, and $n_{I}=n_{1}, n_{R}=n_{2}$.

After some derivation, the $A B C D$ matrix for the tangential and sagittal planes at location $\mathrm{Z}_{3}$, denoted $M_{x}$ and $M_{y}$, respectively, can be written $\operatorname{as}^{20,21}$

$$
\begin{aligned}
& M_{x}= \\
& {\left[\left(\frac{\cos \theta_{2}}{\cos \theta_{1}}\right)+L_{f}\left(\frac{n_{1} \cos \theta_{1}-n_{2} \cos \theta_{2}}{n_{2} R_{f} \cos \theta_{1} \cos \theta_{2}}\right) \quad L_{f}\left(\frac{n_{1} \cos \theta_{1}}{n_{2} \cos \theta_{2}}\right)\right]} \\
& {\left[\begin{array}{ll}
\frac{n_{1} \cos \theta_{1}-n_{2} \cos \theta_{2}}{n_{2} R_{f} \cos \theta_{1} \cos \theta_{2}} & \left(\frac{n_{1} \cos \theta_{1}}{n_{2} \cos \theta_{2}}\right)
\end{array}\right]} \\
& =\left[\begin{array}{ll}
A_{x} & B_{x} \\
C_{x} & D_{x}
\end{array}\right], \\
& M_{y}=\left[\begin{array}{cc}
1+L_{f}\left(\frac{n_{1} \cos \theta_{1}-n_{2} \cos \theta_{2}}{n_{2} R_{f}}\right) & L_{f}\left(\frac{n_{1}}{n_{2}}\right) \\
\frac{n_{1} \cos \theta_{1}-n_{2} \cos \theta_{2}}{n_{2} R_{f}} & \left(\frac{n_{1}}{n_{2}}\right)
\end{array}\right] \\
& =\left[\begin{array}{cc}
A_{y} & B_{y} \\
C_{y} & D_{y}
\end{array}\right],
\end{aligned}
$$

where $n_{1}$ and $n_{2}$ are the refractive indices in the air and fiber and $\theta_{1}$ and $\theta_{2}$ denote the incident and refractive angles, respectively. We assume that $n_{\mathrm{co}} \cong$ $n_{\mathrm{cl}}=n_{2}$; other parameters are defined as

$$
\begin{aligned}
& \theta_{1}=\sin ^{-1}\left(d_{x} / R_{f}\right), \\
& L_{f}=\overline{P Q}=\frac{D_{f}-R_{f}\left(1-\cos \theta_{1}\right)}{\cos \theta_{12}} .
\end{aligned}
$$


The new waist and radius of curvature for the tangential plane $\mathrm{Z}_{3}$ can be expressed as

$$
\begin{aligned}
W_{x 3} & =W_{x 2}\left[\left(A_{x}+\frac{B_{x}}{R_{x 2}}\right)^{2}+\left(\frac{\lambda_{1} B_{x}}{\pi W_{x 2}^{2}}\right)^{2}\right]^{1 / 2}, \\
R_{x 3} & =\frac{\left(A_{x}+\frac{B_{x}}{R_{x 2}}\right)^{2}+\left(\frac{\lambda_{1} B_{x}}{\pi W_{x 2}^{2}}\right)^{2}}{\left(A_{x}+\frac{B_{x}}{R_{x 2}}\right)\left(C_{x}+\frac{D_{x}}{R_{x 2}}\right)+B_{x} D_{x}\left(\frac{\lambda_{1}}{\pi W_{x 2}^{2}}\right)^{2}} .
\end{aligned}
$$

Similarly, we can obtain the waist and the radius of curvature for sagittal plane $\mathrm{Z}_{3}$ by replacing subscript $x$ with $y$. Here we assume Gaussian beam propagation between planes $\mathrm{Z}_{2}$ and $\mathrm{Z}_{3}$.

For the small-angle approximation, the normalized electric field $E^{s}$ of the transformed Gaussian beam expressed in terms of the $X Y Z$ coordinates in plane $\mathrm{Z}_{3}$ is $^{22}$

$$
\begin{aligned}
E^{s}= & \left(\frac{1}{\pi}\right)^{1 / 2}\left(\frac{1}{W_{x 3} W_{y 3}}\right)^{1 / 2} \exp \left\{-\left(\frac{\left(X-d_{x f}\right)^{2}}{W_{x 3}^{2}}+\frac{Y^{2}}{W_{y 3}^{2}}\right)\right. \\
& \left.-j k_{3}\left[\frac{\left(X-d_{x f}\right)^{2}}{2 R_{x 3}}+\frac{Y^{2}}{2 R_{y 3}}+\theta_{12}\left(X-d_{x f}\right)\right]\right\}, \quad 1
\end{aligned}
$$

where $d_{x f}=d_{x}-L_{f} \sin \theta_{12}$ and $k_{3}=2 \pi n_{2} / \lambda_{0}$.

By using the coordinate transformation and assuming that the polarization of the Gaussian beam is in the $Y$ direction, we can express the exact radial and azimuthal vector components of the electric field $\operatorname{as}^{23,24}$

$$
E^{s}(r, \phi)=-j E^{s} \exp (j \phi) \hat{r}+E^{s} \exp (j \phi) \hat{\phi} .
$$

Depending on the radius of the lens, various amounts of the transformed beam will excite the cladding modes, which are then coupled back into the core mode in region III with the aid of a LPFG. Only the excited cladding modes that are phase matched with the grating can be converted efficiently into the core mode at a specific wavelength. Conversely, a part of the transformed beam that excites the core mode will be coupled out into the cladding when it is phase matched. To calculate the coupling efficiency between the transformed Gaussian beam and each fiber mode, we expand the Gaussian beam as

$$
\mathbf{E}^{s}=\sum_{i} a_{i} E_{i}^{\mathrm{co}}+\sum_{v} b_{v} \mathbf{E}_{v}^{\mathrm{cl}}
$$

where the superscripts co and cl denote the core and the cladding modes of a fiber, respectively, and subscripts $i$ and $v$ indicate the kinds of mode. The electric field distribution of each cladding mode can be found in the literature. ${ }^{23}$ The effect of the radiation modes is negligible because of the modes' relatively small values. Core and cladding modes form a complete orthogonal set, and $a_{i}$ and $b_{v}$ are the corresponding coefficients.

The coupling efficiency between the transformed
Gaussian beam and the $v$ th cladding mode is defined as

$\eta_{v}{ }^{s-\mathrm{cl}}=$

power carried by the $v$ th excited cladding mode power carried by the Gaussian beam

$$
=\frac{\frac{1}{2} \operatorname{Re}\left[\left|b_{v}\right|^{2} \iint\left(\mathbf{E}_{v}{ }^{\mathrm{cl}} \times \mathbf{H}_{v}{ }^{\mathrm{cl}}\right) \hat{z} \mathrm{~d} A\right]}{\frac{1}{2} \operatorname{Re}\left[\iint\left(\mathbf{E}^{s} \times \mathbf{H}^{s^{*}}\right) \hat{z} \mathrm{~d} A\right]} .
$$

Similarly, one can obtain the coupling efficiency between the transformed Gaussian beam and the core mode by replacing the superscript cl with co and $b_{v}$ with $a_{i}$.

If the $Z$ component of the electric field is ignored and each fiber mode is normalized, the property of orthogonality requires that any fiber modes $m$ and $n$ $\mathrm{be}^{25}$

$$
\iint \mathbf{E}^{m} \cdot \mathbf{E}^{n^{*} \mathrm{~d} A}=\delta_{m n} .
$$

From Eqs. (14) and (16) we have

$$
\sum_{i}\left|a_{i}\right|^{2}+\sum_{v}\left|b_{v}\right|^{2}=1,
$$

and Eq. (15) can be reduced to

$$
\eta_{v}{ }^{s-\mathrm{cl}}=\left|\iint \mathbf{E}^{s} \cdot \mathbf{E}_{v}^{\mathrm{cl}{ }^{*} \mathrm{~d} A}\right|^{2}=\left|b_{v}\right|^{2} .
$$

Similarly, the coupling efficiency between the transformed Gaussian beam and the $i$ th core mode can be expressed as

$$
\eta_{i}^{s-\mathrm{co}}=\left|\iint \mathbf{E}^{s} \cdot \mathbf{E}_{i}^{\mathrm{co} *} \mathrm{~d} A\right|^{2}=\left|a_{i}\right|^{2} .
$$

According to coupled-mode theory, only the cladding modes that satisfy both phase matching and strong coupling conditions can be efficiently coupled to the core, whereas the others cannot ${ }^{26}$; i.e.,

$$
\begin{gathered}
\lambda_{i}=\lambda_{v} \cong \frac{\left(n^{\mathrm{co}}-n_{v}{ }^{\mathrm{cl}}\right) \Lambda}{\left(1-\frac{\kappa_{01-01}{ }^{\mathrm{c}-\mathrm{co}} \Lambda}{2 \pi}\right)}, \\
{\left[\left(\kappa_{v-01}{ }^{\mathrm{cl}-\mathrm{co}}\right)^{2}+\left(\delta+\frac{\kappa_{01-01}{ }^{\mathrm{co}-\mathrm{co}}}{2}\right)^{2}\right]^{1 / 2} L \cong \frac{\pi}{2},}
\end{gathered}
$$

where $\lambda_{v}$ denotes the $v$ th resonant wavelength. $\quad \lambda_{i}$ is the incident wavelength of the Gaussian beam, $n^{\text {co }}$ and $n_{v}{ }^{\mathrm{cl}}$ are the effective indices of the core mode and the $v$ th cladding mode; $\Lambda$ and $\mathrm{L}$ are the period and the length of the LPFG; and $\kappa_{01-01}{ }^{\text {co-co }}$ and $\kappa_{v-01}{ }^{\text {cl-co }}$ are the coupling coefficients between core-to-core and core-to- $v$ th cladding modes, respectively.

The total coupling efficiency $\eta_{\text {total }}$ is defined as the 
power received inside the core in plane $\mathrm{Z}_{4}$ divided by the total incident power in plane $\mathrm{Z}_{1}$. For a broadband incident beam, $\eta_{\text {total }}$ can be expressed as

$$
\eta_{\text {total }}=\frac{\int P_{i}\left\{\left(\sum_{v}\left(1-S_{v, i}\right)\right) \eta_{i}{ }^{s-\mathrm{co}}+\sum_{v} S_{v, i} \eta_{v, i}{ }^{s-\mathrm{cl}}\right\} \mathrm{d} \lambda_{i}}{\int P_{i} \mathrm{~d} \lambda_{i}},
$$

where $S_{v, i}$ is the transmission loss of the $v$ th cladding mode at wavelength $\lambda_{i}$, which can be solved by use of the coupled-mode theory ${ }^{23} ; \eta_{i}{ }^{s-\mathrm{co}}$ and $\eta_{v, i}{ }^{s-\mathrm{cl}}$ are the coupling efficiency between the transformed Gaussian beam and the fiber core and the $v$ th cladding modes, respectively, at wavelength $\lambda_{i}$, and $P_{i}$ is the optical power density carried by $\lambda_{i}$.

If the Gaussian beam is monochromatic and is set to match a specific transmission wavelength of a LPFG, Eq. (21) is simplified to

$$
\eta_{\text {total }}=\left(1-S_{v}\right) \eta^{s-\mathrm{co}}+S_{v} \eta_{v}^{s-\mathrm{cl}}
$$

Accordingly, we can solve the total coupling efficiency by using Eqs. (12), (18), and (22). Note that our modeling is based on the following assumptions: that (i) a paraxial approximation is made, (ii) the core region in $D_{f}$ is not considered, (iii) the longitudinal electric field is not included, and (iv) backreflection scattering is neglected. To estimate the accuracy of the modeling, the energy conservation is calculated in the reference plane $Z_{3}$ at various working distances as detailed in Appendix A. The result shows that more than $90 \%$ efficiency can be achieved for the working distance within $350 \mu \mathrm{m}$.

\section{Experiment}

Two proofs of experiment are performed to demonstrate the validity of the proposed scheme. In the first experiment, two SMF's of the dispersion-shifted (DS) type are used. The experimental setup is similar to that shown in Fig. 1. SMF1 consists of a LPFG and a lens tip; the output of SMF2 serves as a light source. The far field of SMF2's output has a measured Gaussian fit of $\sim 93.4 \%$. After propagating a working distance $Z_{s}$, the Gaussian beam is transformed by a hemispherical lensed fiber with a radius $R_{f}$. Depending on the radius of the lens, some cladding modes are excited and then coupled into core mode in region III with the aid of a LPFG. The coupling efficiency can be expressed as $P_{\mathrm{SMF} 1} / P_{\mathrm{SMF} 2}$, where $P_{\mathrm{SMF} 1}$ and $P_{\mathrm{SMF} 2}$ are the received and emitted optical power measured from the output ports of SMF1 and SMF2, respectively.

A wavelength-tunable $\mathrm{LD}$ is connected to the input port of SMF2. The wavelength of the $\mathrm{LD}$ is chosen to match that of a LPFG that has minimum transmission. The wavelength of the $\mathrm{LD}$ has a bandwidth of $0.2 \mathrm{~nm}$ at FWHM. A LPFG is imprinted in SMF1 by 248 -nm UV exposure through an amplitude mask of period $450 \mu \mathrm{m}$. SMF1 was previously hydroge-

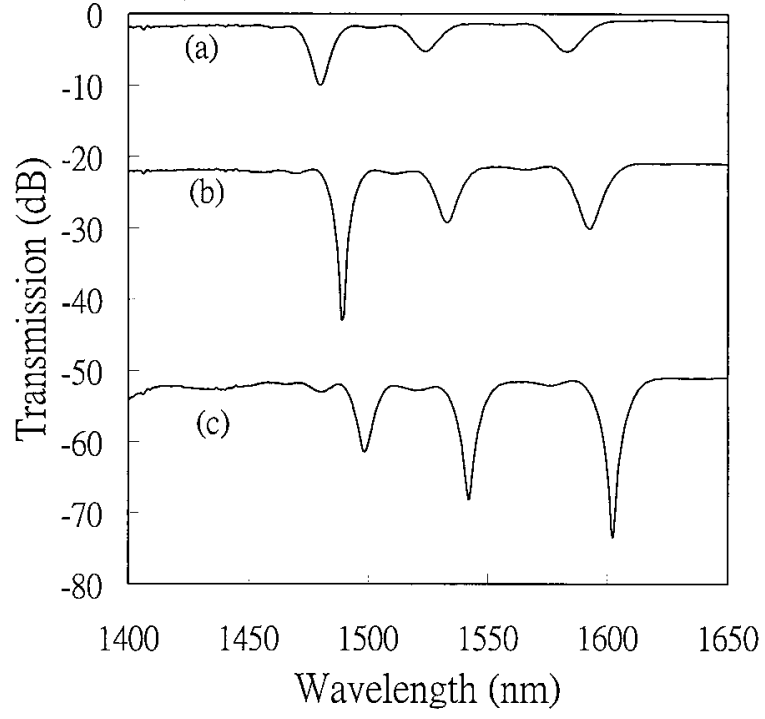

Fig. 2. Evolution of transmission spectra of a LPFG at exposure times (a) $120 \mathrm{~s}$, (b) $180 \mathrm{~s}$, and (c) $210 \mathrm{~s}$.

nated for 15 days at a pressure of $1500 \mathrm{psi}(\sim 77,570$ Torr). The UV exposure area is $\sim 20 \mathrm{~mm}$ in length. Because the electric field profile of the $\mathrm{HE}_{11}$ mode is similar to the Gaussian form, a higher coupling efficiency can be obtained if the imprinted LPFG is properly designed for the $\mathrm{HE}_{11}$ mode. Therefore we need to control the UV-exposure time to obtain the maximum transmission loss for the $\mathrm{HE}_{11}$ cladding mode. The evolution of transmission spectrum of the LPFG is monitored in situ; it is shown in Fig. 2 for three UV exposure times. Note that the UV-exposure time in the middle spectrum of Fig. 2 is a better choice for the desired LPFG. Moreover, note that the transmission spectrum of the DS fiber shows a strong resonant peak at the first cladding mode. The spectrum is different from the step-index spectrum but similar to those reported previously ${ }^{27,28}$ for which two different types of DS fiber were used for LPFG fabrication. The LPFG thus imprinted is then subject to annealing to stabilize its spectral characteristics. SMF1 is cleaved by an electric-arc discharge near the end face of the UV-exposed area to form a hemispherical lens. The radius of curvature can be controlled within a precision of $10 \mu \mathrm{m}$. One puts the two SMF's onto the holders of an automatic alignment setup to search for the maximum coupling power in space at a given working distance.

Figure 3 depicts the variation of coupling efficiency as a function of working distance for a lensed fiber with (solid curve) and without (dashed curve) the LPFG. The corresponding lens radii are 100 and $120 \mu \mathrm{m}$. For a lensed fiber without a LPFG, the coupling efficiency reaches the maximum at a short working distance and then decreases monotonically with the separation. The behavior is similar to butt coupling because of the relatively large radii employed. Conversely, for a lensed fiber with a LPFG, the working distance can be relaxed markedly, with a trade-off in efficiency. For a lens radius of $120 \mu \mathrm{m}$, 


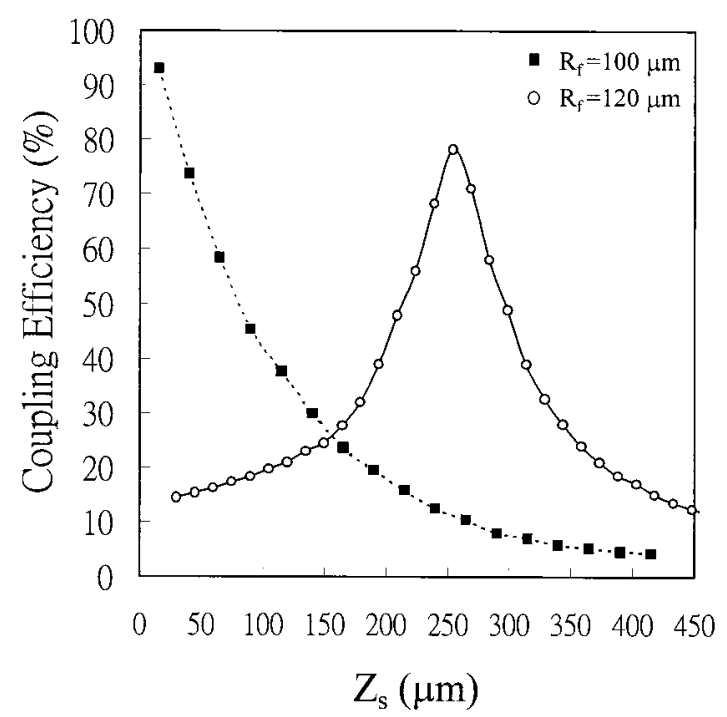

Fig. 3. Coupling efficiency versus working distance for a lensed fiber with (solid curve) and without (dashed curve) the LPFG.

the maximum coupling efficiency is $\sim 78 \%$ at a working distance of $250 \mu \mathrm{m}$, and the corresponding longitudinal tolerance is $\sim 45 \mu \mathrm{m}$ at $1-\mathrm{dB}$ loss. For radii of 100,135 , and $180 \mu \mathrm{m}$, the optimal working distances are 215,300 , and $375 \mu \mathrm{m}$ with reduced coupling efficiencies of $58 \%, 51 \%$, and $43 \%$ and their longitudinal tolerances at $1 \mathrm{~dB}$ loss are $\sim 50,60$, and $90 \mu \mathrm{m}$, respectively. Detailed variation profiles of coupling efficiency as a function of working distance can be found in our earlier paper. ${ }^{15}$ Note that, over such a long range of working distance, only a lensed fiber that contains a LPFG can have a high coupling efficiency. For example, over a working distance of $\sim 210$ to $\sim 300 \mu \mathrm{m}$ the coupling efficiency for the fiber with a $120-\mu \mathrm{m}$-radius lens is more than $50 \%$ and is

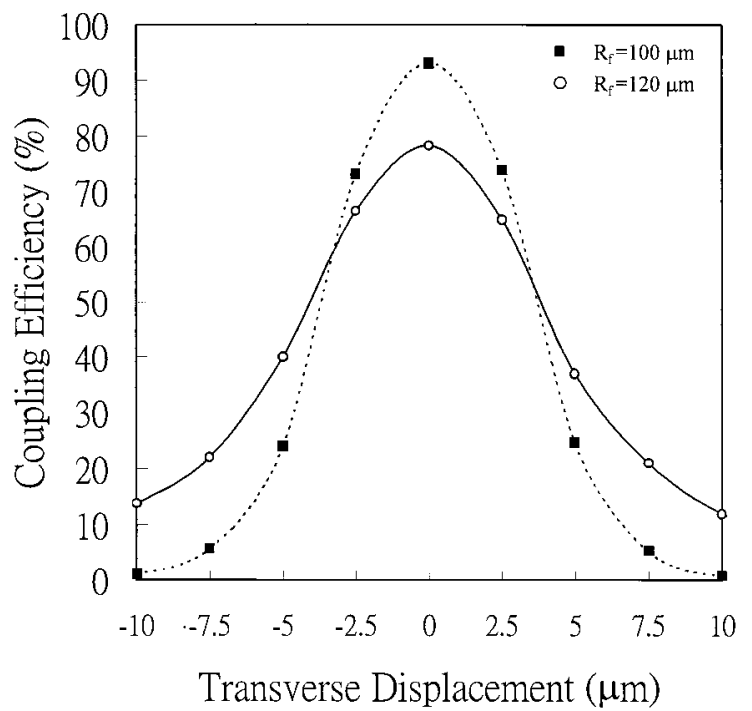

Fig. 4. Coupling efficiency versus transverse displacement for a lensed fiber with (solid curve) and without (dashed curve) the LPFG.

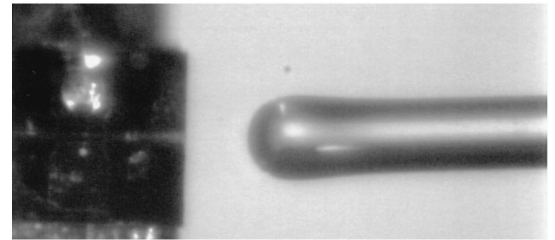

Fig. 5. Microphotograph showing optical coupling between a Fabry-Perot LD and a LPFG lensed fiber.

approximately three to seven times greater than that without the assistance of a LPFG.

Figure 4 shows the variation of coupling efficiency with transverse displacement for a lensed fiber with (solid curve) and without (dashed curve) the LPFG. The corresponding transverse tolerances at $1 \mathrm{~dB}$ loss are $\sim 7.6$ and $\sim 5 \mu \mathrm{m}$, respectively. The other measured transverse tolerances are estimated to be $6.4,9$, and $9 \mu \mathrm{m}$ for the LPFG fibers with radii of 100, 135, and $180 \mu \mathrm{m}$, respectively. All these tolerances are greater than those obtained without the aid of the LPFG.

In the second proof of experiment, a Fabry-Perottype laser diode operating at a wavelength of $1.56 \mu \mathrm{m}$ is used as a light source and replaces SMF2. Its far-field profile is measured in parallel and vertical directions with a beam profiler. The measured beam diameters, defined at $1 / e^{2}$ of the maximum intensity, are 658 and $263 \mu \mathrm{m}$ for the vertical and parallel directions, respectively. As the distance between the end face of LD chip and the sensor head is $470 \mu \mathrm{m}$, the near-field beam waists are estimated to be 0.71 and $1.78 \mu \mathrm{m}$ in the vertical and parallel directions, respectively. The spectral characteristics of the LPFG are adjusted to match the mean wavelength of the LD. Figure 5 is a microphotograph of the optical coupling between a Fabry-Perot LD and a lensed fiber integrated with a LPFG. The lens radius is $70 \mu \mathrm{m}$. The transmission of the LPFG and

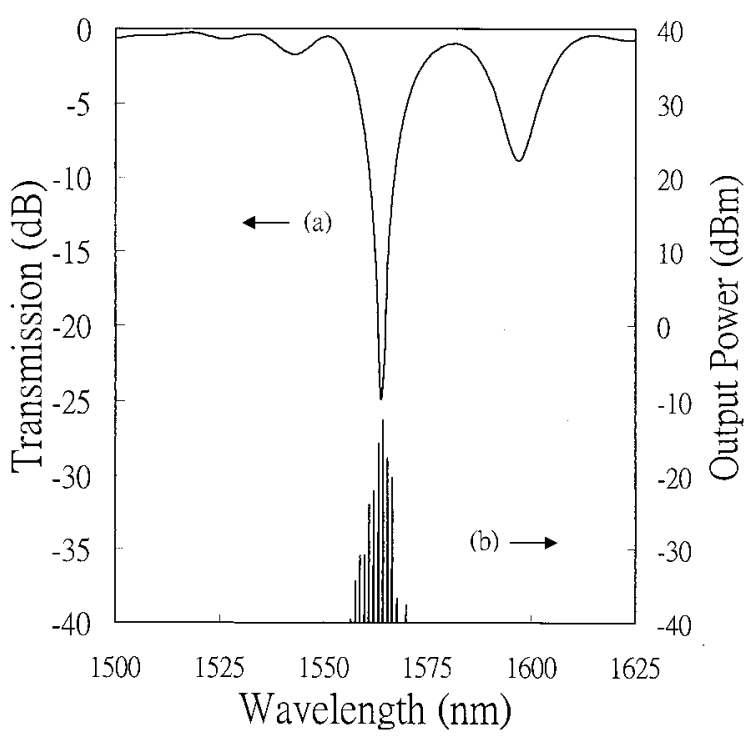

Fig. 6. Spectra of (a) a LPFG and (b) a Fabry-Perot LD. 


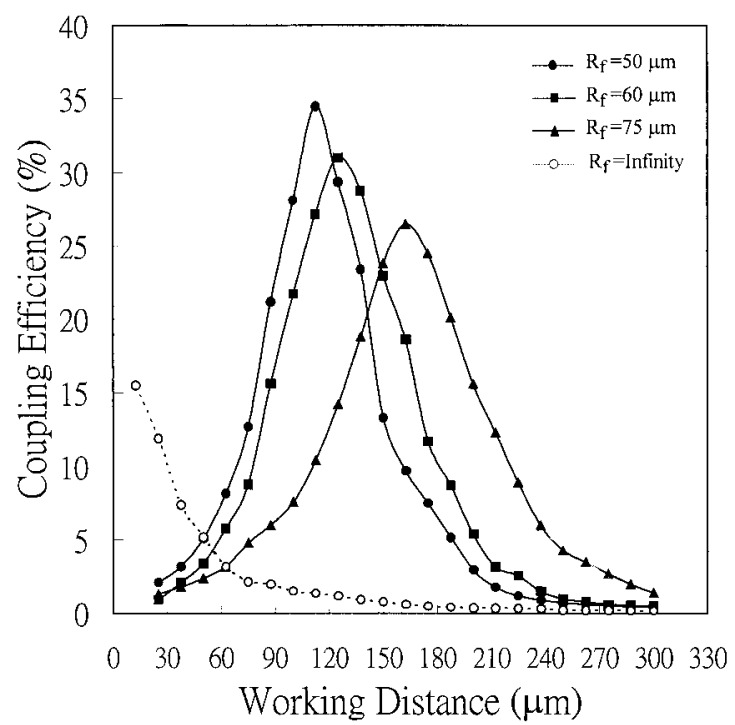

Fig. 7. Measured coupling efficiency versus working distance for lensed fibers with various radii $R_{f}$.

the output spectrum of the Fabry-Perot LD are shown in Fig. 6. The measured 3-dB bandwidth of the LPFG is $\sim 15 \mathrm{~nm}$, larger than the $\sim 1 \mathrm{~nm}$ of the LD.

Figure 7 depicts coupling efficiency versus working distance for several radii. The dashed and solid curves correspond to cases of butt coupling (i.e., infinite $R_{f}$ and without LPFG) and the lensed fiber integrated with a LPFG, respectively. In the case of butt coupling, the maximum coupling efficiency is $\sim 15.5 \%$ at a working distance of $\sim 12 \mu \mathrm{m}$ and then decreases monotonically with separation. Conversely, for a lensed fiber integrated with a LPGF, at such a short working distance only $\sim 1.5 \%$ coupling efficiency is measured; however, the efficiency increases to its maximum at a markedly long working distance. For example, for a lens radius of $50 \mu \mathrm{m}$, the maximum coupling efficiency is $\sim 34.5 \%$ at a working distance of $110 \mu \mathrm{m}$, and the corresponding longitudinal tolerance is $\sim 26 \mu \mathrm{m}$ at 1 -dB loss. For other radii, of 60 and $75 \mu \mathrm{m}$, the optimal working distances are 125 and $160 \mu \mathrm{m}$ with corresponding coupling efficiencies of $31 \%$ and $26 \%$, respectively. Their longitudinal tolerances at 1-dB loss are $\sim 40$ and $\sim 50 \mu \mathrm{m}$, respectively. Note that, over such a long range of working distance, only the lensed fiber with LPFG could have a high coupling efficiency. For example, over a working distance of $\sim 85$ to $\sim 140$ $\mu \mathrm{m}$ the LPFG with a $50-\mu \mathrm{m}$-radius lens can have $\sim 10$ to $\sim 20$ times greater coupling efficiency than that of butt coupling. The transverse tolerances at $1-\mathrm{dB}$ loss of their peak efficiencies are measured as 2.6, 2.8, and $3.2 \mu \mathrm{m}$ for fibers with radii of 50,60 , and $75 \mu \mathrm{m}$, respectively. For comparison, the measured buttcoupling tolerances are $\sim 10$ and $\sim 2 \mu \mathrm{m}$ for longitudinal and transverse directions, respectively, at 1-dB loss.

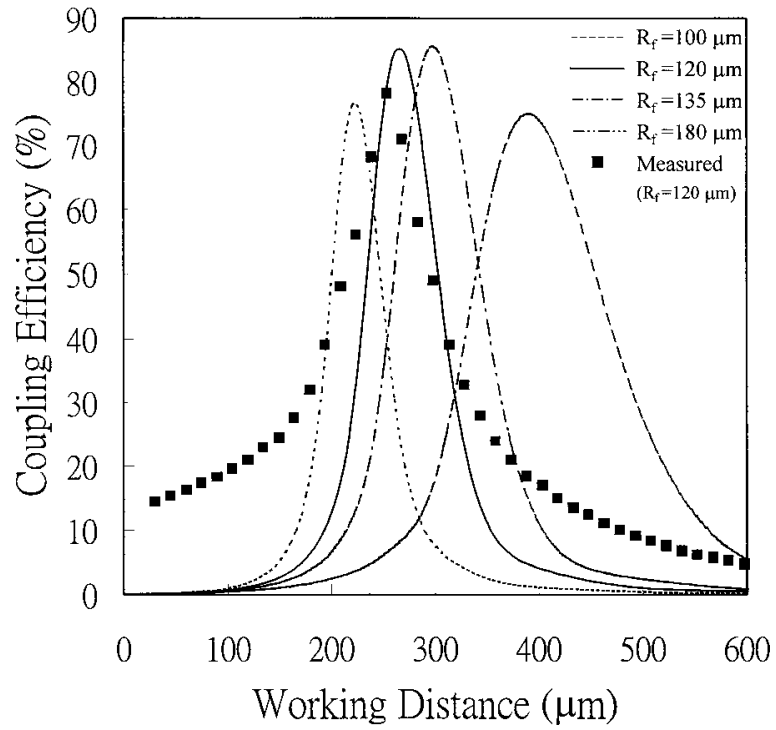

Fig. 8. Simulated coupling efficiency versus working distance for a lensed fiber with various radii $R_{f}$. Also shown is the measured result for $R_{f}=120 \mu \mathrm{m}$.

\section{Discussion}

In this section we first compare the measured results with the simulated results based on the theory described in Section 2. Then, based on further approximations, we derive the optimal working distance and lens radius. Moreover, we discuss some important considerations when the proposed new scheme is practically applied.

\section{A. Simulation Results}

The radial and azimuthal electric field of a fiber cladding mode can be obtained by solution of the problem of a three-layer, step-index, weakly guiding SMF subjected to the boundary conditions at each interface. ${ }^{23}$ The following parameters are assumed: index of core, $n_{\mathrm{co}}=1.458$; index of cladding, $n_{\mathrm{cl}}=1.45$; radius of core, $a_{\mathrm{co}}=2.75 \mu \mathrm{m}$; radius of cladding, $a_{\mathrm{cl}}=62.5$ $\mu \mathrm{m}$; and mode field diameter of incident Gaussian beam, $W_{0}=6.5 \mu \mathrm{m}$. Thus the total coupling efficiency as a function of working distance can be solved numerically with Eqs. (12), (18), and (22). Figure 8 shows the simulation results for various radii of lensed fibers in an ideal case; i.e., when the transmission of $\mathrm{HE}_{11}$ of the LPFG is zero. Here we ignore the thickness $D_{f}$ of the lensed fiber. The calculated maximum coupling efficiencies are found to be approximately $76 \%, 86 \%, 85 \%$, and $75 \%$ for $R_{f}$ of 100 , 120,135 , and $180 \mu \mathrm{m}$, respectively. The estimated corresponding longitudinal tolerances at $1 \mathrm{~dB}$ are approximately $35,45,55$, and $85 \mu \mathrm{m}$, which are similar to the measured tolerances. However, there are some discrepancies between the simulated and measured results. First, all simulated results show narrower tolerances, especially at the tail ends. Second, the calculated maximum coupling efficiencies differ from the measured ones. These results can be attributed to the following situations: (1) The 


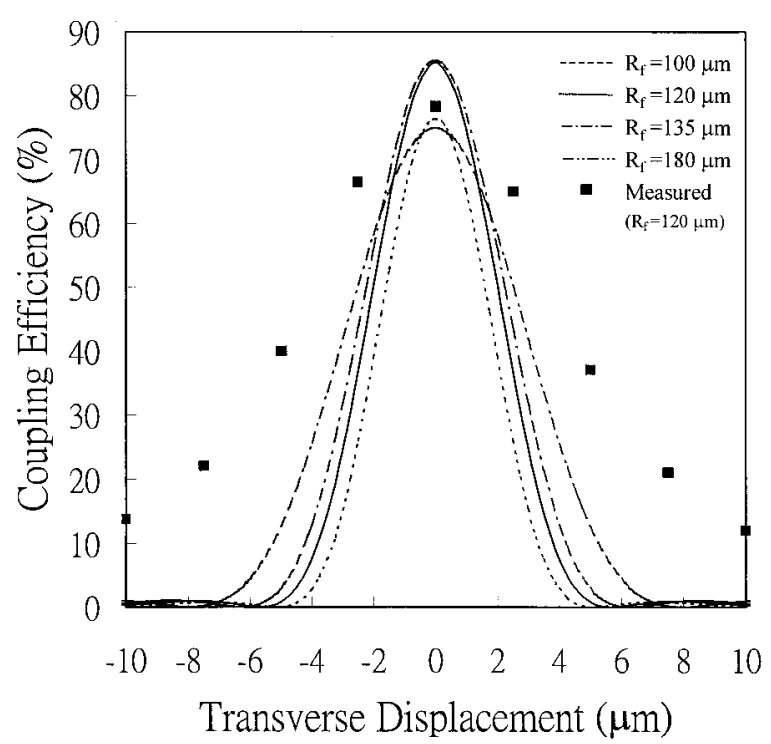

Fig. 9. Simulated coupling efficiency versus transverse displacement for a lensed fiber with various radii $R_{f}$. Also shown is the measured result for $R_{f}=120 \mu \mathrm{m}$.

index profile of the DS fiber used in the experiment has a shape that is closer to that of a triangle than to a step shape as used in the simulation; therefore the actual electrical field distribution is different from the simulated one. (2) The bending is induced during the assembly of the LPFG; therefore the transmission loss of the LPFG will be strongly reduced and the resonant peak will be shifted to the longerwavelength side. (3) We ignore the existence of the core in region II of our model and find that the transformed Gaussian beam may be slightly distorted. The reason for our not using a common step-index fiber for the experiment is that the transmission of the $\mathrm{HE}_{11}$ mode thus fabricated is relatively weak, only -1 to $-2 \mathrm{~dB}$ for a strong LPFG. ${ }^{23}$ Moreover, we observe that the $\mathrm{HE}_{11}$ mode of a LPFG made from a DS fiber is almost insensitive to the surrounding medium, a property that makes further recoating of the fiber easier.

Figure 9 shows simulated coupling efficiency versus transverse displacement for lensed fibers with various radii. The simulated transverse tolerances at $3-\mathrm{dB}$ loss for $R_{f}=100,120,135,180 \mu \mathrm{m}$ are approximately $4,4.5,5$, and $6.5 \mu \mathrm{m}$, respectively. The measured transverse tolerance is larger than the simulated tolerance for the same reasons as described above.

\section{B. Optimal Working Distance}

An approximate working distance of the $v$ th cladding mode for the maximum coupling efficiency, defined as $Z_{s, \text { optm }}{ }^{v}$, can be derived if the radius of the lensed fiber is given. Using Eqs. (6), (7), (10), and (11) and assuming that $D_{f}=d_{x}=0$, we have

$$
R_{x 3}=\frac{n_{\mathrm{cl}} R_{f} R_{2}}{n_{1} R_{f}-R_{2}\left(n_{\mathrm{cl}}-n_{1}\right)}
$$

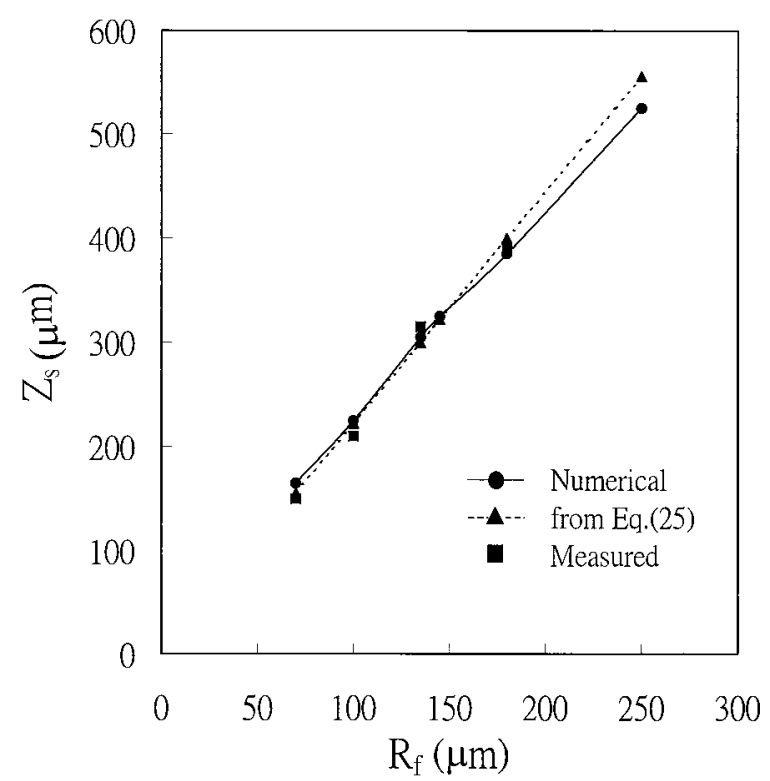

Fig. 10. Variation of optimal working distance with radii of a lensed fiber.

$$
W_{x 3}=W_{x 2} .
$$

Based on the ray optics approximation, the guided angle for the $v$ th cladding mode can be estimated as $\theta_{v}{ }^{\mathrm{cl}}=(\pi / 2)-\theta_{3}=\cos ^{-1}\left(n_{1 v}^{\mathrm{cl}} / n_{\mathrm{cl}}\right)$, where $n_{1 v}{ }^{\mathrm{cl}} \mathrm{de}-$ notes the effective index of the $v$ th cladding mode. To obtain an analytical solution for the optimal working distance we assume that $\theta_{v}{ }^{\mathrm{cl}} \sim \pm W_{x 3} / R_{x 3}$, where + and - correspond to divergent and convergent wave fronts, respectively. By using Eqs. (2), (3), and (23) as well as assuming that $Z_{s} / Z_{0} \gg 1$, we can approximate the optimal working distance for the $v$ th cladding mode as

$$
\begin{aligned}
& Z_{s, \mathrm{optm}}{ }^{v} \approx\left(\frac{R_{f}}{n_{\mathrm{cl}}-n_{1}}\right)\left[n_{1} \pm \frac{\pi W_{0} n_{\mathrm{cl}} \theta_{v}{ }^{\mathrm{cl}}}{\lambda_{i}}\right], \\
& Z_{s, \mathrm{optm}}{ }^{1} \approx \frac{n_{1} R_{f}}{n_{\mathrm{cl}}-n_{1}} .
\end{aligned}
$$

Expression (25) is obtained for the $\mathrm{HE}_{11}$ mode because the magnitude of $\theta_{1}{ }^{\mathrm{cl}}$ is very small $(\sim \pm 0.00872$ $\operatorname{rad}$ for $\left.\lambda_{i}=1.51 \mu \mathrm{m}\right)$. Therefore the working distance for the maximum coupling efficiency increases linearly with the lens radius, as shown in Fig. 10, in which the approximate result is seen to be similar to those from numerical and measured calculations. The optimal working distance of the $\mathrm{HE}_{11}$ mode is located near the focal plane of the hemispherical lens, implying that the wave front that is characteristic of a $\mathrm{HE}_{11}$ mode is approximately a plane wave.

\section{Optimal Lens Radius}

It is important to find the optimal radius of a lensed fiber for the maximum coupling efficiency when the LPFG parameters, wavelength, and mode field diameter of the incident Gaussian beam are given. Unfortunately, there is no analytical solution. For 


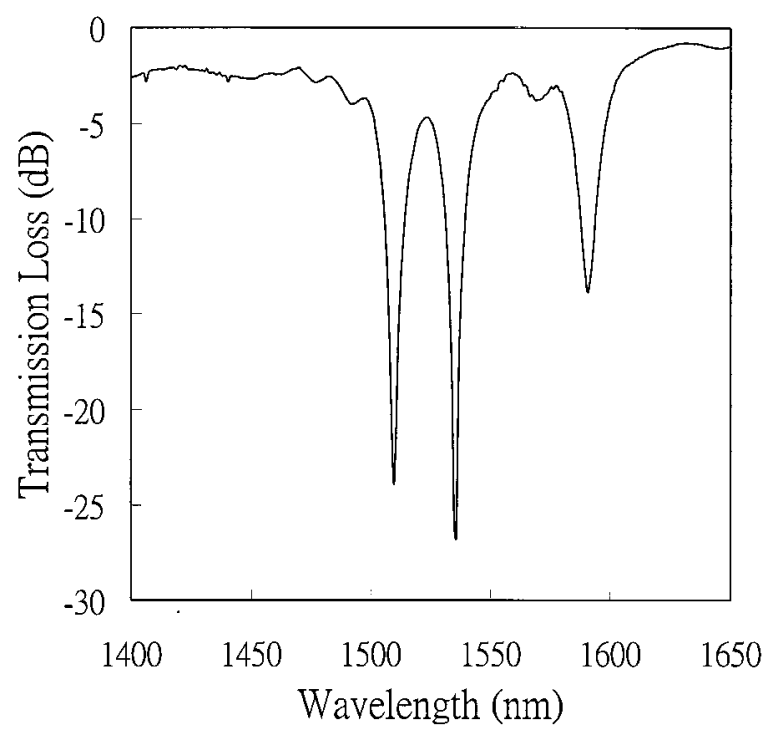

Fig. 11. Transmission spectrum of the LPFG used for coupling of the $\mathrm{HE}_{11}$ and $\mathrm{HE}_{13}$ modes.

estimation, we assume only that the transformed Gaussian beam that satisfies the following condition can lead to the maximum coupling for the $\mathrm{HE}_{11}$ mode:

$$
W_{3, \mathrm{optm}}=\mathrm{Cr}_{\max }^{\mathrm{cl}},
$$

where $W_{3, \text { optm }}$ is the waist of the transformed Gaussian beam at the optimal working distance $Z_{s, 0 p t m}{ }^{1}$ for the optimal lens radius; $r_{\max }{ }_{\text {cl }}$ is the radial position of the maximum magnitude of the $\mathrm{HE}_{11}$ cladding mode obtained by definition of a three-layer step-index $\mathrm{SMF}$, and $C$ is an adjustable parameter. Therefore, from Eqs. (2) and (23) and expression (25), the optimal radius $R_{f, \text { optm }}{ }^{11}$ can be expressed as

$$
R_{f, \mathrm{optm}}{ }^{11}=\frac{C \pi W_{0} r_{\max }{ }^{\mathrm{cl}}\left(n_{\mathrm{cl}}-n_{1}\right)}{n_{1} \lambda_{1}},
$$

where $W_{0}$ and $\lambda_{i}$ are the waist and the wavelength, respectively, of the incident Gaussian beam. Using the same parameters and $r_{\max }{ }^{\mathrm{cl}}=26 \mu \mathrm{m}$ and assuming that $W_{3, \text { optm }} \approx 1.8 r_{\text {max }}{ }^{\text {cl }}$, we have $R_{f, \text { optm }}{ }^{11} \sim 140$, $48 \mu \mathrm{m}$ for SMF-to-SMF and LD-to-SMF coupling, respectively. The results are consistent with the calculated ones, i.e., $R_{f, \text { optm }}{ }^{11} \sim 138,45 \mu \mathrm{m}$, which we obtained by numerically solving Eqs. (12), (18), and (22) for various radii $R_{f}^{11}$.

\section{Mode Dependence}

To distinguish the coupling efficiencies that resulted from $\mathrm{HE}_{11}$ and $\mathrm{HE}_{13}$ modes we use a fiber with a radius of curvature of $\sim 145 \mu \mathrm{m}$ and the transmission spectrum in Fig. 11. For measurement of coupling efficiencies, the laser diode is tuned to match the resonant wavelengths of the $L P F G$, i.e., $\lambda_{i}=1510 \mathrm{~nm}$ and $\lambda_{i}=1536 \mathrm{~nm}$ for $\mathrm{HE}_{11}$ and $\mathrm{HE}_{13}$, respectively. The experimental relationship of coupling efficiency to working distance is shown in Fig. 12. Note there are two maxima of working distances (250 and 425 $\mu \mathrm{m})$ for the $\mathrm{HE}_{13}$ mode but only one maximum $(\sim 325$

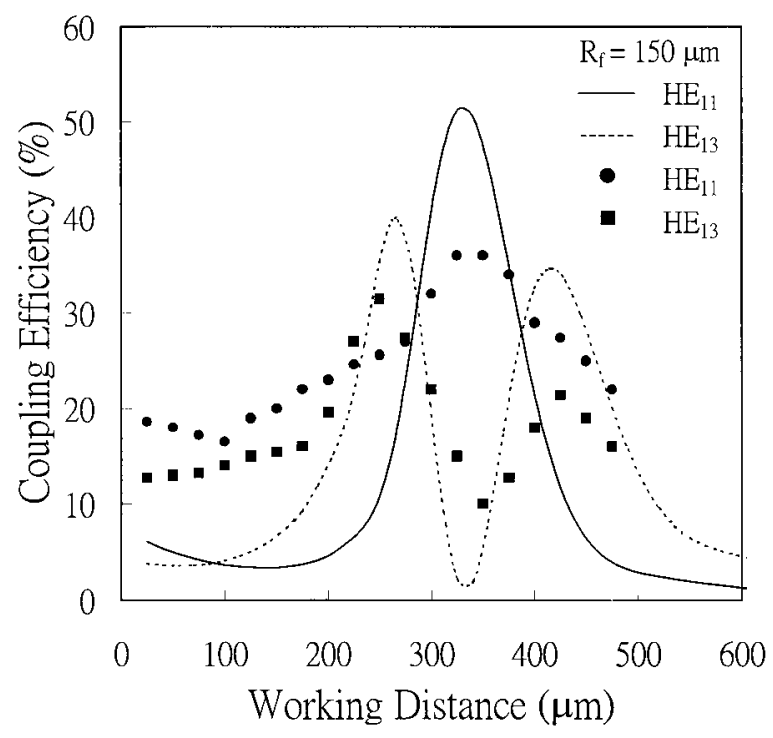

Fig. 12. Measured and simulated coupling efficiencies of $\mathrm{HE}_{11}$ and $\mathrm{HE}_{13}$ modes versus working distance.

$\mu \mathrm{m})$ for the $\mathrm{HE}_{11}$ mode. The coupling efficiency shows a linear decay only for the $\mathrm{HE}_{11}$ mode but not for the $\mathrm{HE}_{13}$ mode at small working distances, perhaps because of a relatively weak loss for the $\mathrm{HE}_{11}$ mode. Therefore a portion of beam is directly coupled into the core and remains propagating in the core at shorter working distances.

Also shown in Fig. 12 is the relationship of simulated coupling efficiency to working distance for the $\mathrm{HE}_{11}$ and $\mathrm{HE}_{13}$ cladding modes. The parameters remain the same, except that losses $S$ are assumed to be 0.65 and 0.8 for modes $\mathrm{HE}_{11}$ and $\mathrm{HE}_{13}$, respectively. There are two local maxima of coupling efficiency for the $\mathrm{HE}_{13}$ mode. Note that the location of the maximum coupling efficiency of the $\mathrm{HE}_{11}$ mode is exactly the same as that of the local minimum of the $\mathrm{HE}_{13}$ mode, which can be understood from the law of energy conservation even though the resonant $\mathrm{HE}_{11}$ and $\mathrm{HE}_{13}$ wavelengths are slightly different. Because most of the energy of the incident Gaussian beam is transformed into mode $\mathrm{HE}_{11}$ at plane $\mathrm{Z}_{3}$ at the optimal working distance $Z_{s, \text { optm }}{ }^{1}$, no other cladding modes will be excited. The simulated longitudinal and transverse tolerances at 1-dB loss are estimated to be approximately $20-25$ and $1.5-1.8$ $\mu \mathrm{m}$, respectively, for $\mathrm{HE}_{13}$ coupling. The location of the maximum coupling efficiency for $\mathrm{HE}_{13}$ can be estimated from expression (24). The calculated effective index $n_{13}$ cl of the $\mathrm{HE}_{13}$ mode is 1.4497 for $\lambda_{i}=$ $1.53 \mu \mathrm{m}$, which we obtain by modeling the fiber as a three-layer step-index structure, and its corresponding guided angle is $1.048^{\circ}$. Therefore the locations of maximum coupling efficiency are $\sim 280$ and $\sim 390$ $\mu \mathrm{m}$. However, as shown in Fig. 12, the corresponding measured locations are $\sim 265$ and $\sim 415 \mu \mathrm{m}$. The difference is relatively larger than for the $\mathrm{HE}_{11}$ mode because the guided angle of $\mathrm{HE}_{13}$ is twice as long as that of $\mathrm{HE}_{11}$. Although the approximate re- 


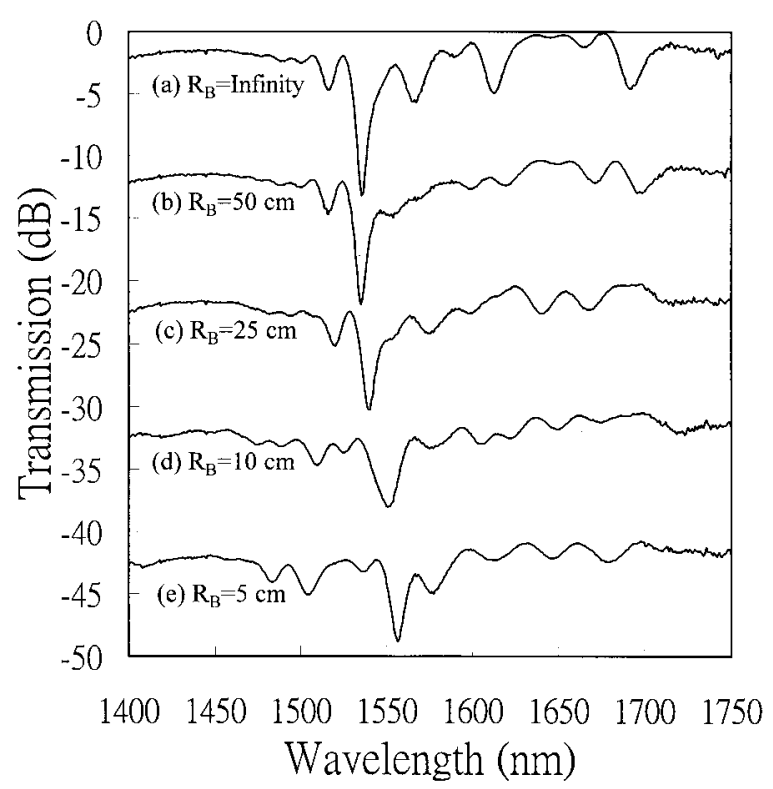

Fig. 13. Evolution of transmission spectra at various bend radii $R_{B}$.

sult is slightly different from the simulated one, it still provides a good first-order estimation.

\section{E. Bending Effect}

Because the transmission spectrum of a LPFG is highly sensitive to external bending, the resonant peak of the LPFG will shift to the longer-wavelength side and the transmission loss will change drastically. Figure 13 shows the experiment results for various bending radii $R_{B}$. Therefore it is important to avoid excess bending on a LPFG in the practical packaging process when such a scheme is applied for optical coupling.

\section{F. Wavelength and Temperature Dependence}

Inasmuch as only the modes that satisfy the phasematching condition can be efficiently coupled into the core, the proposed coupling scheme is obviously wavelength dependent. Figure 14 depicts the dependence of coupling efficiency on wavelength at the optimal working distance, i.e., $Z_{s}=250 \mu \mathrm{m}$, and the loss spectrum of the integrated LPFG. It can be seen that the coupling efficiency almost follows the loss spectrum of the LPFG, which can be understood from Eq. (22) because the coupling efficiency $\eta_{\text {total }}$ is almost proportional to the LPFG loss $S_{v}$ if $\eta_{v}{ }^{s-\mathrm{cl}}$ is larger than $\eta^{s-c o}$. The ratio of $\eta_{1}{ }^{s-c l}$ at the maximum peak, i.e., $\lambda=1535 \mathrm{~nm}$, is estimated to be $84 \%$, which is close to the theoretical value of $88 \%$. In general, a LPFG is temperature sensitive; thus the phase-matching condition may change with the environment, resulting in variations of coupling efficiency. The problem can be solved, for example, by use of a novel temperature-insensitive long-period fiber grating. ${ }^{29}$

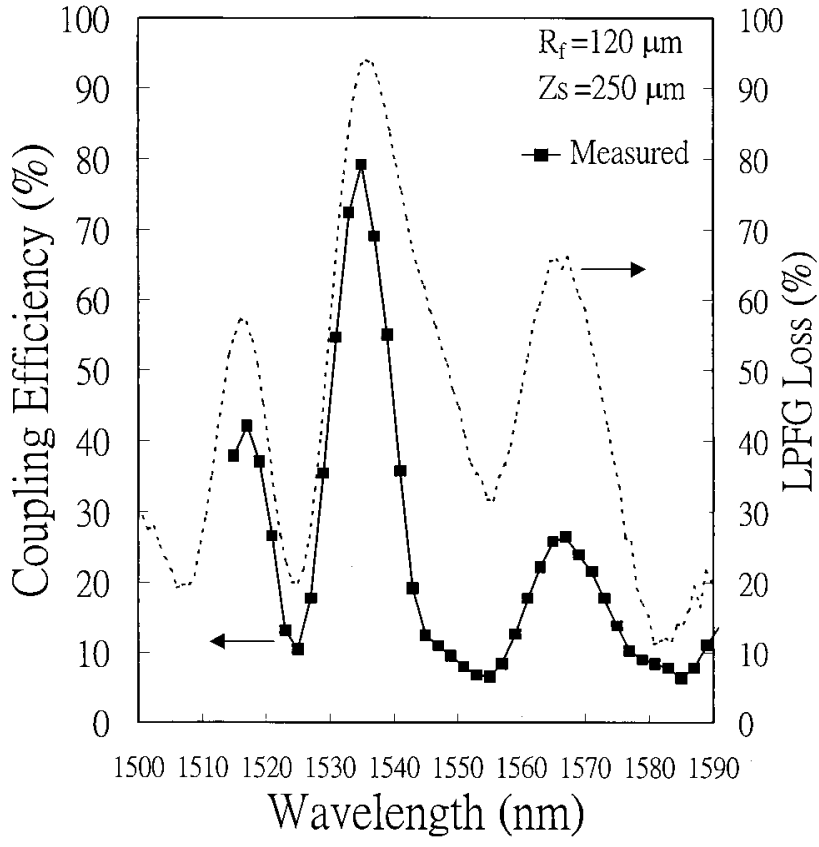

Fig. 14. Measured dependence of coupling efficiency and loss of LPFG (dashed curve) on wavelength.

\section{Conclusions}

A lensed fiber is considered more stable and compact than a nonlensed fiber in optical coupling. However, in most cases it is practically difficult to assemble and mass produce a lensed fiber owing to its small working distance and critical lens radius. Here we propose a novel optical coupling scheme that uses a hemispherical lensed fiber integrated with a LPFG. The proposed novel coupling scheme was demonstrated in both SMF-to-SMF and LD-to-SMF proofs of experiment. A coupling efficiency of $\sim 78 \%$ was obtained with a lens radius of $\sim 120 \mu \mathrm{m}$ at a working distance of $\sim 250 \mu \mathrm{m}$ when the wavelength of the incident Gaussian beam was tuned to match the resonant wavelength of the $\mathrm{HE}_{11}$ cladding mode. As for $\mathrm{HE}_{13}$ coupling, two working distances that lead to the maxima of coupling efficiency were found.

We employed a model based on an $A B C D$ matrix and mode expansion to calculate the coupling efficiency at various working distances. The results show that (1) utilization of the novel coupling scheme results in a long working distance because most of the launched beam is coupled to the cladding rather to the core, (2) maximum efficiency can be obtained in $\mathrm{HE}_{11}$ coupling because of the similar Gaussian profile but not in other cladding modes, and (3) the calculated working distance and the longitudinal tolerance for the maximum coupling efficiency are consistent with measured ones for both $\mathrm{HE}_{11}$ and $\mathrm{HE}_{13}$ coupling.

Based on a ray optics approximation, an optimal working distance and an optimal lens radius were derived for the maximum coupling efficiency. These approximate results are in good agreement with the calculated and measured ones. Achieving the max- 


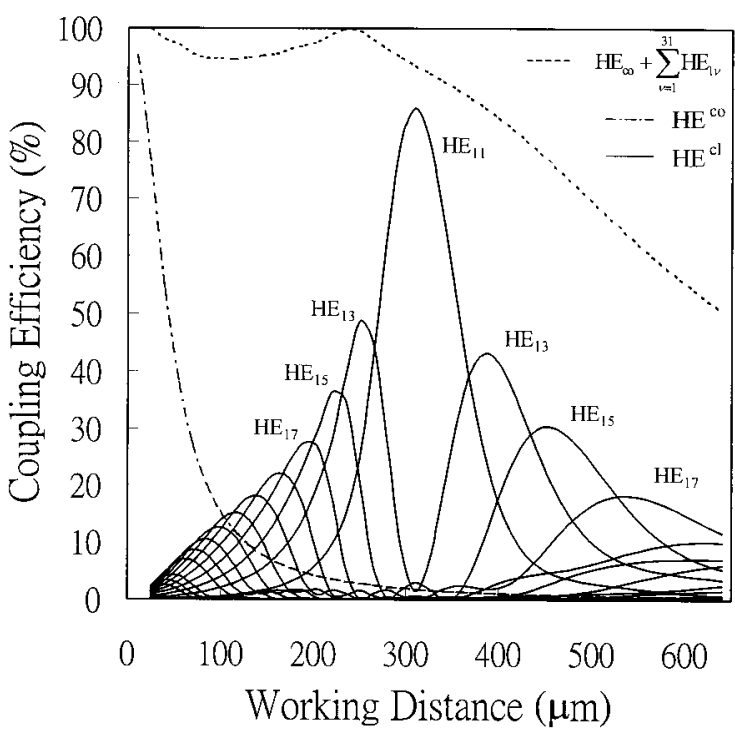

Fig. 15. Simulated coupling efficiencies and their sum versus working distance for various cladding and core modes.

imum coupling efficiency requires that the following conditions be fulfilled: (i) $\mathrm{HE}_{11}$ coupling is preferred, (ii) the lens radius should be matched to Eq. (27), (iii) the Gaussian beam waist should be positioned as directed by expression (25), (iv) the wavelength between the incident Gaussian beam and the resonant peak of the $\mathrm{HE}_{11}$ mode should be matched, (v) the transmission loss for the $\mathrm{HE}_{11}$ mode should be as strong as possible, and (vi) external bending and a wavelength-shift effect should be avoided in the practical application.

\section{Appendix A}

In Section 2 we assume that the transformed Gaussian beam can be expanded by use of a complete set derived from a three-layer fiber mode. As shown by Eqs. (14)-(18), the total coupling efficiency $\eta_{\text {total }}$, defined as $\eta_{\text {total }}=\sum_{v=1}^{v=\infty} \eta^{s-c o}+\eta_{v}{ }^{s-c l}$, should be equal to unity for any working distance from the viewpoint of energy conservation. To estimate the accuracy of our model, we discuss the energy conservation at reference plane $\mathrm{Z}_{3}$ for various working distances. The radius of curvature is chosen to be $140 \mu \mathrm{m}$ because there the simulated coupling efficiency becomes maximum, as mentioned in Subsection 4.C. Other parameters are the same as described in Section 4, except that $D_{f}=0$ and $S=0,1$ for the core and the cladding modes, respectively. Figure 15 shows the simulated coupling efficiency versus working distance for various even cladding modes and a core mode. The odd cladding modes are not shown because of their relatively weak magnitudes. The total coupling efficiency $\eta_{\text {total }}$ is obtained from the first 16 even cladding modes. That the total coupling efficiency is more than $90 \%$ as the working distance is $350 \mu \mathrm{m}$ or less but decreases almost linearly as the working distance increases. The waist of Gaussian beam is $\sim 56 \mu \mathrm{m}$ in plane $Z_{3}$ for $Z_{s}=350 \mu \mathrm{m}$ and becomes $\sim 96 \mu \mathrm{m}$ for $Z_{s}=600 \mu \mathrm{m}$. As the working distance becomes greater than $350 \mu \mathrm{m}$, the beam size exceeds the fiber's diameter and causes the total coupling efficiency to decrease; a portion of the Gaussian beam could become radiation modes and escape from the cladding-air interface. Note that there is still a discrepancy between the simulated result and the ideal one, i.e., $\eta_{\text {total }}=1$, possibly because of the finite superposition of the cladding modes.

The authors thank S. H. Tseng and G. W. Chern for their assistance in the calculation of the three-layer fiber mode, C. D. Su for helpful discussions, and the Industrial Technology Research Institute/OptoElectronics and System Laboratories for providing the beam profiler. The authors are grateful for partial support by the National Science Council, Taiwan, under contract NSC 89-2215-E-002-013 and by the Education Ministry of Taiwan under contract 89-EFA06-2-4.

\section{References}

1. A. M. Vengsakar, P. J. Lemaire, J. B. Judkins, V. Bhatia, T. Erdogan, and J. Sipe, "Long-period fiber gratings as bandrejection filters," J. Lightwave Technol. 4, 58-65 (1996).

2. A. M. Vengsakar, J. R. Pedrazzani, J. B. Judkins, and P. J. Lemaire, "Long-period fiber-grating-based gain equalizers," Opt. Lett. 21, 336-338 (1996).

3. D. C. Johnson, F. Bilodeau, B. Malo, K. O. Hill, P. G. J. Wigley, and G. I. Stegeman, "Long-length, long-period rocking filters fabricated from conventional monomode telecommunications optical fiber," Opt. Lett. 17, 1635-1637 (1992).

4. K. O. Hill, B. Malo, K. A. Vineberg, F. Bilodeau, D. C. Johnson, and I. Skinner, "Efficient mode conversion in telecommunication fiber using externally written gratings," Electron. Lett. 26, 1270-1272 (1990).

5. V. Bhatia and A. M. Vengsarkar, "Optical fiber long-period grating sensors," Opt. Lett. 21, 692-694 (1996).

6. J. Yamada, Y. Murakami, J. Sakai, and T. Kimura, "Characteristics of a hemispherical microlens for coupling between a semiconductor laser and single-mode fiber," IEEE J. Quantum. Electron. QE-16, 1067-1072 (1980).

7. H. Kuwahara, M. Sasaki, and N. Tokoyo, "Efficient coupling from semiconductor lasers into single-mode fibers with tapered hemispherical ends," J. Lightwave Technol. 14, 58-65 (1996).

8. C. Edwards, H. Presby, and C. Dragone, "Ideal microlenses for laser to fiber coupling," J. Lightwave Technol. 11, 252-257 (1993).

9. W. L. Emkey and C. A. Jack, "Analysis and evaluation of graded-index fiber-lenses," J. Lightwave Technol. 5, 11561164 (1987).

10. K. Shiraishi, "A lensed fiber with a long working distance for integrated coupling between laser diodes and single-mode fibers," J. Lightwave Technol. 13, 1736-1744 (1995).

11. W. Bludau and R. H. Rosserg, "Low-loss laser-to-fiber coupling with negligible optical feedback," J. Lightwave Technol. LT-3, 294-302 (1985).

12. I. Moerman, P. P. Van Daele, and P. M. Demeester, "A review on fabrication technologies for the monolithic integration of tapers with III-V semiconductor devices," IEEE J. Sel. Top. Quantum. Electron. 3, 1308-1320 (1997).

13. N. Kalonji and J. Semo, "High efficiency long working distance laser diode to singlemode fiber coupling arrangement," Electron. Lett. 30, 892-893 (1994).

14. P. Chanclou, M. Thual, J. Lostec, P. Auvray, J. Caulet, G. Joulié, A. Poudoulec, and B. Clavel, "Highly efficient collective 
coupling between laser diode array and lensed fiber ribbon," Electron. Lett. 34, 273-274 (1998).

15. W. T. Chen and L. A. Wang, "Optical coupling between singlemode fibers by utilizing long-period fibre grating," Electron. Lett. 35, 421-423 (1999).

16. H. J. Patrick, C. G. Askins, R. W. McElhanon, and E. J. Friebel, "Amplitude mask patterned on an excimer laser mirror for high intensity writing of long period fiber gratings," Electron. Lett. 33, 1167-1168 (1997).

17. E. M. Dianove, D. S. Stardubov, S. A. Vasiliev, A. A. Frolov, and O. I. Medvedkov, "Refractive-index gratings written by near-ultraviolet radiation," Opt. Lett. 22, 221-223 (1997).

18. D. D. Davis, T. K. Gaylord, E. N. Glytsis, and S. C. Mettle, " $\mathrm{CO}_{2}$ laser-induced long-period fiber gratings: spectral characteristics, cladding modes and polarisation independence," Electron. Lett. 34, 1416-1417 (1998).

19. T. Enomoto, M. Shigehara, S. Ishikawa, T. Danzuka, and H. Kanamori, "Long-period fiber grating in a pure-silica-core fiber written by residual stress relaxation," in Optical Fiber Communication Conference (OFC), Vol. 2 of 1998 OSA Technical Digest Series (Optical Society of America, Washington, D.C., 1998), paper ThG2.

20. H. Kogelnik, "On the propagation of Gaussian beams of light through lenslike media including those with a loss or gain variation," Appl. Opt. 4, 1562-1569 (1965).

21. G. A. Massey and A. E. Siegman, "Reflection and refraction of a Gaussian light beam at tilted ellipsoidal surfaces," Appl. Opt. 8, 975-978 (1969).

22. J.-I. Sakai and T. Kimura, "Design of a miniature lens for semiconductor laser to single-mode fiber coupling," IEEE J. Quantum Electron. QE-16, 1059-1066 (1980).

23. T. Erdogan, "Cladding-mode resonances in short- and longperiod fiber grating filters," J. Opt. Soc. Am. A 14, 1760-1773 (1997).

24. D. Marcuse, Theory of Dielectric Optical Waveguides (Academic, Boston, Mass., 1991), Chaps. 2 and 3.

25. A. Yariv and P. Yeh, Optical Waves in Crystals (Wiley, New York, 1983), Chap. 11.

26. T. Erdogan, "Fiber grating spectra," J. Lightwave Technol. 15, 1277-1294 (1997).

27. H. J. Patrick, A. D. Kersey, and F. Bucholtz, "Analysis of the response of long period fiber grating to external index of refraction,” J. Lightwave Technol. 16, 1606-1611 (1998).

28. B. H. Lee and J. Nishii, "Cladding-surrounding interface insensitive long-period grating," Electron. Lett. 34, 1129-1130 (1998).

29. K. Shima, K. Himeno, T. Sakai, S. Okude, A. Wada, and R. Yamauchi, "A novel temperature-insensitive long-period fiber grating using a boron-codoped-germanosilicate-core fiber," in Optical Fiber Communication Conference, Vol. 6 of 1997 OSA Technical Digest Series (Optical Society of America, Washington, D.C., 1997), paper FB2. 